\title{
Clinical outcomes among hemodialysis patients with atrial fibrillation: a Korean nationwide population-based study
}

\author{
Yeunmi Kang ${ }^{1}$, Hyung Yun $\mathrm{Choi}^{2}$, Young Eun Kwon ${ }^{3}$, Ji Hyeon Shin ${ }^{4}$, Eun Mi Won ${ }^{4}$, Ki Hwa Yang ${ }^{4}$, \\ Hyung Jung $\mathrm{Oh}^{5,6}$, Dong-Ryeol Ryu ${ }^{6,7}$ \\ ${ }^{1}$ Department of Internal Medicine, Ewha Womans University Mokdong Hospital, Seoul, Republic of Korea \\ ${ }^{2}$ The Korean Society of Nephrology, Seoul, Republic of Korea \\ ${ }^{3}$ Department of Internal Medicine, Myongji Hospital, Hanyang University College of Medicine, Goyang, Republic of Korea \\ ${ }^{4}$ Health Insurance Review and Assessment Service, Wonju, Republic of Korea \\ ${ }^{5}$ Ewha Institute of Convergence Medicine, Ewha Womans University Mokdong Hospital, Seoul, Republic of Korea \\ ${ }^{6}$ Research Institute for Human Health Information, Ewha Womans University Mokdong Hospital, Seoul, Republic of Korea \\ ${ }^{7}$ Department of Internal Medicine, Ewha Womans University School of Medicine, Seoul, Republic of Korea
}

Background: The number of patients requiring dialysis is increasing worldwide, and the atrial fibrillation and atrial flutter (AF) prevalence among hemodialysis (HD) patients is higher than in the general population. There have been no studies of Korean AF patients undergoing HD that investigated how AF affects outcomes, such as all-cause mortality, hospitalization, and stroke events. We conducted a large-scale retrospective cohort study with data from the National Health Insurance System to determine how AF affects these outcomes.

Methods: In 2013, the Health Insurance Review and Assessment service, a Korean national health insurance scheme, collected data from 21,839 HD patients to evaluate the adequacy of dialysis centers. All-cause mortality, hospitalization, and stroke events were compared between patients with and without AF. Sub-analyses compared these outcomes between AF patients receiving warfarin and those not receiving warfarin.

Results: Cox regression analysis found that AF was a significant risk factor for death from any cause (hazard ratio [HR], 1.356; 95\% confidence interval [Cl], 1.222-1.506; $p$ < 0.001), hospitalization (HR, 1.323; 95\% Cl, 1.225-1.430; $p<0.001)$, and hemorrhagic stroke (HR, 1.500; 95\% Cl, 1.050-2.141; $p=0.026)$. AF was not significantly associated with an increased risk of ischemic stroke. The use of warfarin was significantly associated with hemorrhagic stroke incidence (HR, 1.593; 95\% Cl, 1.075-2.360; $p=0.020)$, while there was no significant correlation between warfarin treatment and all-cause mortality, hospitalization, and ischemic stroke. Conclusion: This cohort study of Korean dialysis patients showed that AF was a risk factor for multiple outcomes among HD patients.

Keywords: Atrial fibrillation, Hemodialysis, Hemorrhagic stroke, Hospitalization, Ischemic stroke, Mortality, Warfarin

Received: February 3, 2020; Revised: July 30, 2020; Accepted: September 10, 2020

Editor: Eun Hui Bae, Chonnam National University, Gwangju, Republic of Korea

Correspondence:

Hyung Jung Oh

Ewha Institute of Convergence Medicine, Ewha Womans University Mokdong Hospital, 1071 Anyangcheon-ro, Yangcheon-gu, Seoul 07985, Republic of Korea. E-mail: ohjmd@naver.com

ORCID: https://orcid.org/0000-0002-4281-696X

Dong-Ryeol Ryu

Department of Internal Medicine, Ewha Womans University School of Medicine, 260 Gonghang-daero, Gangseo-gu, Seoul 07804, Republic of Korea. E-mail: drryu@ewha.ac.kr

Copyright (c) 2021 by The Korean Society of Nephrology

(a) This is an Open Access article distributed under the terms of the Creative Commons Attribution Non-Commercial and No Derivatives License (http:// creativecommons.org/licenses/by-nc-nd/4.0/) which permits unrestricted non-commercial use, distribution of the material without any modifications, and reproduction in any medium, provided the original works properly cited. 


\section{Introduction}

Atrial fibrillation and atrial flutter (AF) is the most common arrhythmia in the general population, and its prevalence among end-stage renal disease (ESRD) patients undergoing hemodialysis (HD) is higher (ranges from $3.8 \%$ to $27 \%$ ) [1] than that in general population (ranges from $2.5 \%$ to $3.5 \%$ ) $[2,3]$. Moreover, the incidence of AF increases with age and dialysis duration among chronic HD patients [4]. AF commonly arises secondarily to comorbid conditions, such as coronary artery disease, pericarditis, mitral valve disease, cardiomyopathy, and HD itself, causes fluid swings that lead to heart failure and left atrial dilatation $[5,6]$. Thus, the occurrence of AF among chronic HD patients may carry a poor prognosis [7]. To the best of our knowledge, there have been no studies investigating the impact of $\mathrm{AF}$ on adverse clinical outcomes among Korean chronic HD patients.

Since AF is well known as a major cause of ischemic stroke in the general population, most physicians consider prescribing anticoagulants for patients at high risk of ischemic stroke. Likewise, among AF patients in the general population, anticoagulant therapy, usually with warfarin, has been used to prevent ischemic stroke among chronic HD patients. However, warfarin should be administered with caution because it can increase bleeding. Additionally, chronic HD patients require special care when taking warfarin because they are more likely to be at risk of bleeding. Thus, routine warfarin administration for chronic HD patients is not considered standard treatment. Moreover, AF in chronic HD patients likely does not increase the risk of stroke compared with AF in the general population. Therefore, Kidney Disease Improving Global Outcomes (KDIGO) guidelines do not advise AF patients to take anticoagulation agents routinely to prevent ischemic stroke when they are undergoing chronic HD [8]. In contrast, the American Heart Association/American College of Cardiology/Heart Rhythm Society (AHA/ACC/ HRS) recommends warfarin administration for the HD population, similar to that for the general population [9].

This study aimed to investigate the impact of AF on adverse clinical outcomes (all-cause mortality, hospitalization, and ischemic or hemorrhagic stroke) among a large population of maintenance HD patients in Korea. Moreover, we examined the effects of warfarin use on clinical outcomes among AF patients undergoing chronic HD.

\section{Methods}

\section{Study population and data collection}

The National Health Insurance System (NHIS) in Korea is a single-payer system to which $98 \%$ of citizens belong. Under this system, medical providers request reimbursements from the NHIS. This nationwide cohort study relies on data from the Korean Health Insurance Review and Assessment (HIRA). We used the claim data between January 2013 and December 2017. Among members of the general population who used medical services during the period, we chose patients who were undergoing chronic HD. Chronic HD patients were identified by the International Classification of Disease, 10th revision (ICD-10) diagnoses and hospital billing records (O7020, O9991) for at least 90 days. Adults over 18 years who had been receiving conventional maintenance $\mathrm{HD}$ for at least 3 months were eligible for this study.

There is a regular national assessment for dialysis adequacy in Korean medical institutions where HD has been performed, and we enrolled a total number of 21,839 patients who were assessed for dialysis adequacy in 2013. Fig. 1 shows the process of subject selection. Between October and December 2013, a total of 53,607 patients under HD were charged with HD claims (O7020, O9991). During the same period, 50,397 patients with chronic HD including inpatient and outpatient were sorted. After removing patients who met the exclusion criteria, 21,839 patients were classified as subjects for the adequacy assessment.

There were a total of 735 institutions requesting adequacy assessment; 10 were excluded and 725 were evaluated. The criteria for selecting an institution to be evaluated were institutions that possess HD machines as of October 1, 2013 and those that produced HD billing codes (O7020, O9991). Conditions for exclusions included institutions that did not have an outpatient undergoing HD or was closed at the time of December 2013. Therefore, from October to December 2013, among the 735 dialysis centers that had HD machines and claimed medical codes related to HD, 725 centers (98.6\%) were assessed.

We chose the following data because the follow-up duration of patients of the 2013 adequacy assessment was longer than that of the 2015 adequacy assessment. Baseline data, including demographics, comorbid diseases, and laboratory 


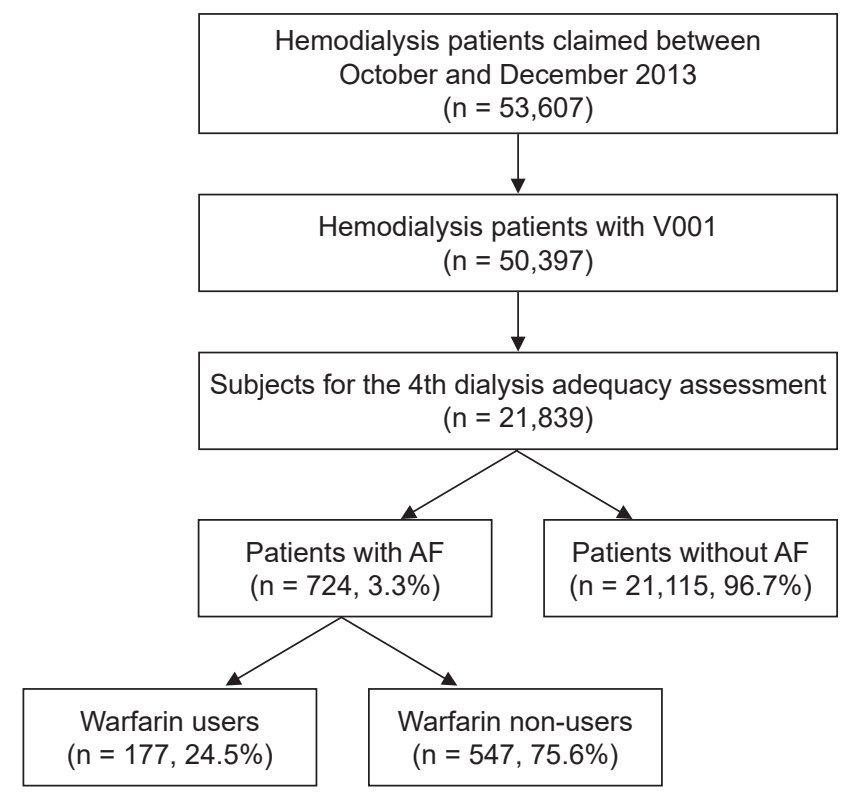

Figure. 1. Study flow diagram. From October to December 2013, the number of patients who were treated with hemodialysis as inpatient or outpatient claimed with fee code for hemodialysis (07020, 09991). Among 53,607, the number of patients with V001 code. This code is limited to the patients who were treated with dialysis over 90 days for chronic kidney disease. The final number of subjects for the 4th dialysis adequacy assessment is 21,839 . The exclusion criteria are 1 ) hemodialysis less than twice a week, 2) hemodialysis less than 90 days, or 3) age less than 18 years.

$\mathrm{AF}$, atrial fibrillation and atrial flutter.

data, were collected at the time of patient enrollment. In the case of comorbid diseases, ICD-10 code of principal and secondary diagnosis from January 1, 2013 to December 31, 2013 was extracted as the comorbid disease if there was a hospitalization or outpatient medical history. Comorbidities shown in Table 1 and Supplementary Table 1 (available online) included 17 conditions of Charlson comorbidity index (CCI) and a few more conditions (Supplementary Table 2, 3; available online). We calculated the CCI scores according to CCI standards (Supplementary Table 2).

The current study was performed in accordance with the Declaration of Helsinki 2013 and approved by the Institutional Review Board (IRB) of Ewha Womans University, Mokdong Hospital (No. EUMC 2018-12-25). As the study subjects were deidentified, the IRB waived the need for written consent from the patients.

\section{Definitions}

AF patients were identified as patients diagnosed with I48 according to ICD-10 code as a principle or as secondary disease among 10 coexisting diseases between January and December 2013. We also included subjects as AF patients who answered "yes" to the questionnaire for dialysis assessment asking if he/she used medication or kept a pacemaker because of AF.

All-cause mortality was regarded as death if a person was extracted from enrollment in the HIRA, and 'hospitalization' was considered when a patient had been hospitalized at least once during the follow-up period from January 1 , 2014 to December 31, 2017. All-cause hospitalization was identified.

An ischemic stroke event was recognized when a subject was hospitalized with I63-, I65-, or I66- as a principal disease during the period from January 1, 2014 to December 31, 2017 or when diagnosed with these codes as coexisting disease during that period if there was no such illness in 2013. Up to 10 coexisting diseases were searched. Likewise, a hemorrhagic stroke event was identified when a patient had been hospitalized with I60.-, I61.- or I62.- during the same follow-up period or when diagnosed with a coexisting disease during that period, if there was no such disease in 2013.

Warfarin users were defined as those who had $>90$ days of cumulative prescriptions for warfarin in 2013. The drug ingredient codes were 249105ATB and 249103ATB. Additionally, comorbid diseases were identified from the list of ICD-10 codes if there was a hospitalization or outpatient history of the disease during the period of 2013.

\section{Outcomes}

Primary outcomes were determined as all-cause mortality, hospitalization, and stroke events that occurred from January 1, 2014 to December 31, 2017.

\section{Statistical analysis}

We first stratified the total enrolled HD patients into two groups based on the existence of $\mathrm{AF}$ (AF vs. non-AF) to investigate the impact of AF on clinical outcomes. Next, we classified AF patients into two groups based on the use of 
Table 1. Baseline patient characteristics

\begin{tabular}{|c|c|c|c|c|}
\hline Variable & All $(n=21,839)$ & Non-AF $(n=21,115)$ & $\mathrm{AF}(\mathrm{n}=724)$ & p-value \\
\hline Age (yr) & $59.3 \pm 13.0$ & $59.1 \pm 13.0$ & $64.0 \pm 11.0$ & $<0.001$ \\
\hline Male sex & $12,766(58.5)$ & $12,304(58.3)$ & $462(63.8)$ & 0.003 \\
\hline Body mass index $\left(\mathrm{kg} / \mathrm{m}^{2}\right)$ & $22.2 \pm 3.3$ & $22.2 \pm 3.3$ & $22.1 \pm 3.2$ & 0.582 \\
\hline SBP, pre-HD (mmHg) & $141.2 \pm 15.9$ & $141.3 \pm 15.9$ & $138.1 \pm 15.4$ & $<0.0001$ \\
\hline DBP, pre-HD (mmHg) & $78.8 \pm 9.4$ & $78.8 \pm 9.4$ & $76.9 \pm 9.4$ & $<0.0001$ \\
\hline $\mathrm{DM}$ & $8,831(40.4)$ & 8,579 (40.6) & $252(34.8)$ & \\
\hline HTN & $5,906(27.0)$ & $5,656(26.8)$ & $250(34.5)$ & \\
\hline CGN & 2,603 (11.9) & $2,535(12.0)$ & $68(9.4)$ & \\
\hline Others & $1,859(8.5)$ & $1,799(8.5)$ & $60(8.3)$ & \\
\hline Unknown & $2,640(12.1)$ & $2,546(12.1)$ & $94(13.0)$ & \\
\hline Creatinine (mg/dL) & $9.7 \pm 2.8$ & $9.7 \pm 2.7$ & $9.2 \pm 2.6$ & $<0.0001$ \\
\hline Total calcium (mg/dL) & $9.0 \pm 0.9$ & $9.0 \pm 0.9$ & $9.0 \pm 0.8$ & 0.220 \\
\hline Phosphorus (mg/dL) & $5.1 \pm 1.4$ & $5.1 \pm 1.4$ & $4.9 \pm 1.3$ & 0.001 \\
\hline Urea reduction ratio (\%) & $70.7 \pm 6.0$ & $70.8 \pm 6.0$ & $70.5 \pm 5.7$ & 0.200 \\
\hline \multicolumn{5}{|l|}{ Comorbidity } \\
\hline Acute MI & $410(1.9)$ & 377 (1.8) & $33(4.6)$ & $<0.0001$ \\
\hline $\mathrm{CHF}$ & 995 (4.6) & $886(4.2)$ & 109 (15.1) & $<0.0001$ \\
\hline PVD & $983(4.5)$ & $942(4.5)$ & $41(5.7)$ & 0.125 \\
\hline Cerebrovascular disease & $1,485(6.8)$ & $1,400(6.6)$ & $85(11.7)$ & $<0.0001$ \\
\hline Dementia & $649(3.0)$ & $627(3.0)$ & $22(3.0)$ & 0.914 \\
\hline DM with complication & $7,690(35.2)$ & $7,442(35.3)$ & $248(34.3)$ & 0.583 \\
\hline Hemiparesis & $163(0.7)$ & $155(0.7)$ & $8(1.1)$ & 0.254 \\
\hline Chronic renal disease & $21,835(100)$ & $21,115(100)$ & $724(100)$ & 0.711 \\
\hline Cancer without metastasis & $1,253(5.7)$ & $1,206(5.7)$ & $47(6.5)$ & 0.375 \\
\hline Cancer with metastasis & $48(0.2)$ & $46(0.2)$ & $2(0.3)$ & 0.674 \\
\hline AIDS & $1(0.0)$ & $1(0.0)$ & $0(0)$ & 0.999 \\
\hline HTN & $11,594(53.1)$ & $11,142(52.8)$ & $452(62.4)$ & $<0.0001$ \\
\hline DM & $9,649(44.2)$ & $9,329(44.2)$ & $320(44.2)$ & 0.993 \\
\hline Ischemic heart disease & $3,163(14.5)$ & $2,922(13.8)$ & 241 (33.3) & $<0.0001$ \\
\hline Stroke & $1,258(5.8)$ & 1,186 (5.6) & 73 (10.1) & $<0.0001$ \\
\hline Cancer & $1,257(5.8)$ & $1,210(5.7)$ & $47(6.5)$ & 0.387 \\
\hline $\mathrm{AF}$ & 724 (3.3) & NA & NA & NA \\
\hline
\end{tabular}

Data are expressed as mean \pm standard deviation or number (\%).

$\mathrm{AF}$, atrial fibrillation and atrial flutter; AIDS, acquired immunodeficiency syndrome; CGN, chronic glomerulonephritis; CHF, congestive heart failure; DBP, diastolic blood pressure; DM, diabetes mellitus; ESRD, end-stage renal disease; HD, hemodialysis; HTN, hypertension; MI, myocardial infarction; NA, not available; PVD, peripheral vascular disease; SBP, systolic blood pressure. 
warfarin (warfarin user vs. warfarin non-user) to examine the effect of warfarin use on clinical outcomes among AF patients undergoing HD.

To compare baseline characteristics, chi-square tests for categorical variables and unpaired t-tests for continuous variables were performed. Continuous variables were expressed as means and standard deviations, and categorical variables were described using frequencies and percentages. Cox proportional hazard modeling was used to explore the association between $\mathrm{AF}$ and the incidence of outcomes; all-cause mortality, hospitalization, and stroke events (ischemic or hemorrhagic). Moreover, as the CCI includes common comorbidities, we used it to adjust for potentially confounding variables. Additionally, Cox proportional hazard models were conducted to reveal the impact of warfarin on the occurrence of adverse clinical outcomes.

The cumulative event-free survival rates were estimated by Kaplan-Meier analysis and log-rank test. This was used to compare chronic $\mathrm{HD}$ patients with $\mathrm{AF}$ and without $\mathrm{AF}$ with respect to event rates of new ischemic stroke, hemorrhagic stroke, hospitalization, or all-cause mortality. Survival time was defined as the interval between time at the beginning of cohort and the onset of the endpoint of follow-up, whichever came first. Repeated events were not considered.

All statistical tests were evaluated using a two-tailed 95\% confidence interval (CI) with hazard ratios (HRs). Age, sex, body mass index (BMI), systolic blood pressure (SBP), diastolic blood pressure (DBP), and CCI were used for the adjusted variables. All analyses were performed using SAS, version 9.4 (SAS Institute, Cary, NC, USA), and $\mathrm{p}<0.05$ was considered statistically significant.

\section{Results}

\section{Baseline characteristics}

Table 1 shows the baseline characteristics among enrolled patients. In the total patient group $(\mathrm{n}=21,839)$, the mean age was $59.3 \pm 13.0$ years; 12,766 patients $(58.5 \%)$ were men; the mean dialysis vintage was $5.6 \pm 5.1$ years; the mean BMI was $22.2 \pm 3.3 \mathrm{~kg} / \mathrm{m}^{2}$; and the mean SBP and DBP were 141.2 \pm 15.9 and $78.8 \pm 15.9 \mathrm{mmHg}$, respectively. Among the total patients, 724 patients (3.3\%) were identified with AF (Table 1).

The patients with AF were significantly older, more likely to be male, had been undergoing HD for longer, and had higher mean SBP and DBP than those without AF. There were also significantly more several comorbid diseases, such as acute myocardial infarction, congestive heart failure, cerebrovascular disease, chronic pulmonary disease, peptic ulcer, and hypertension, in the AF group compared with the non-AF group, while there was no significant difference in urea reduction ratio or the prevalence of diabetes mellitus (DM) between groups (Table 1).

Effects of atrial fibrillation on hemodialysis patients: allcause mortality, hospitalization, and stroke events

Multivariate Cox proportional regression analysis model 1 showed that AF was a significant risk factor for all-cause mortality (HR, 1.356; 95\% CI, 1.222-1.506; p < 0.001), hospitalization (HR, 1.323; 95\% CI, 1.225-1.430, $\mathrm{p}<0.001$ ), and hemorrhagic stroke (HR, 1.500; 95\% CI, 1.050-2.141; $\mathrm{p}=0.026$ ), after adjusting for age, sex, BMI, SBP, DBP, and CCI. However, AF was not significantly associated with an increased risk of ischemic stroke (HR, 1.240; 95\% CI, 0.9611.601; $\mathrm{p}=0.984$ ) (Table 2). Multivariate Cox proportional regression analysis model 2 , which was selectively adjusted with dialysis vintage, albumin, acute myocardial infarction, congestive heart failure, cerebrovascular disease, and hypertension, also showed that AF was a significant risk factor for all-cause mortality (HR, 1.294; 95\% CI, 1.152-1.452, $\mathrm{p}<0.001$ ), hospitalization (HR, 1.276; 95\% CI, 1.171-1.390, $\mathrm{p}<0.001$ ), and hemorrhagic stroke (HR, 1.766; 95\% CI, 1.211-2.576; $\mathrm{p}=0.003$ ). In model 2, similar to model 1, AF was not significantly associated with an increased risk of ischemic stroke (HR, 1.182; 95\% CI, 0.888-1.574; $\mathrm{p}=$ 0.251) (Table 3). The findings of univariate Cox proportional regression analyses are presented in Supplementary Table 4 (available online).

The mortality, hospitalization, cerebral infarction, and cerebral hemorrhage by absence vs. presence of AF were estimated through Kaplan-Meier curve analysis. The rates for all four outcomes were higher in the AF group; the differences were statistically significant, with a p-value less than 0.05 (Supplementary Fig. 1, available online).

\section{Effects of warfarin treatment on atrial fibrillation patients undergoing hemodialysis}

We next explored the impact of warfarin use on clinical 

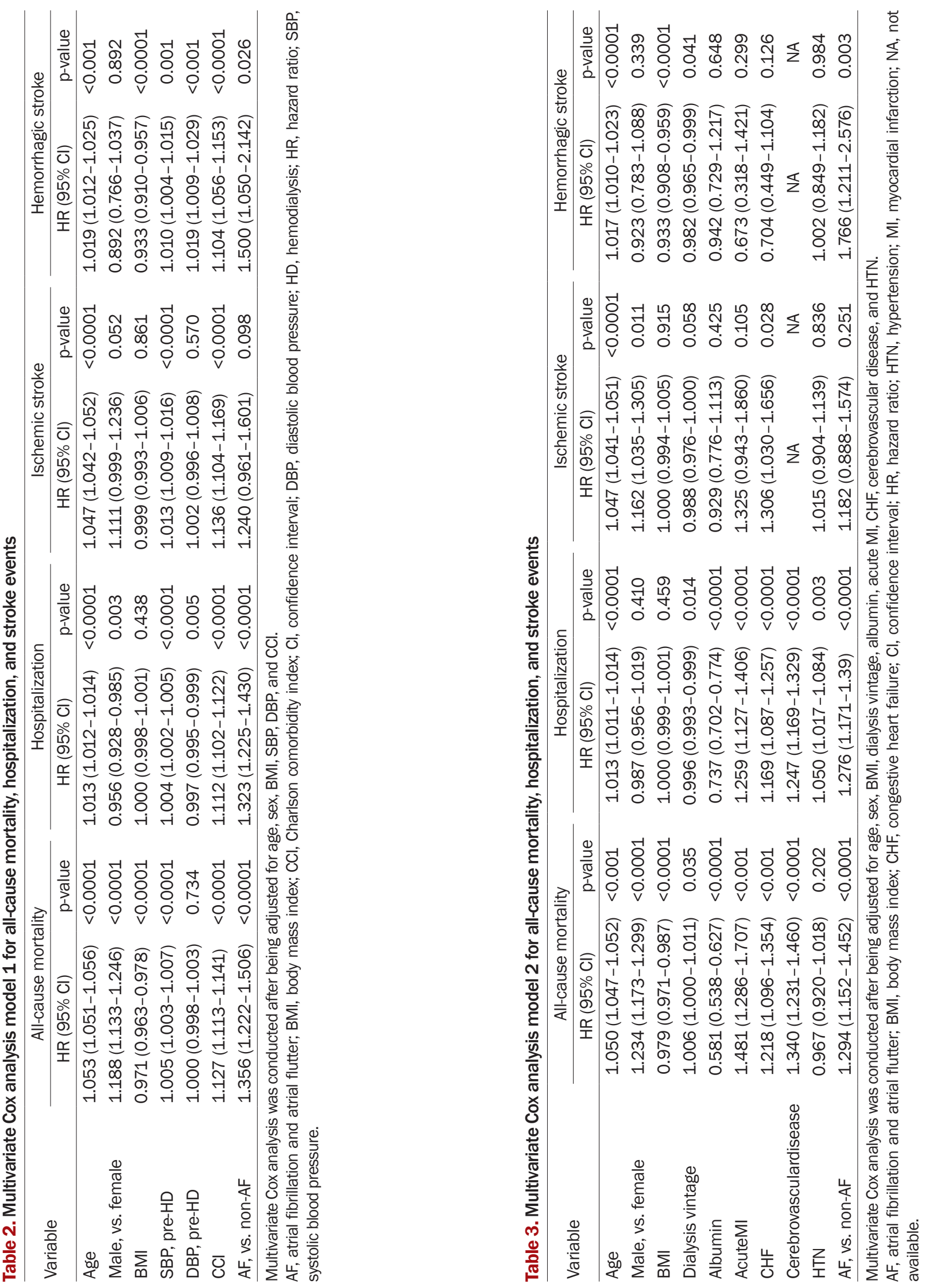
outcomes (all-cause mortality, hospitalization, and ischemic or hemorrhagic stroke) among AF patients undergoing $\mathrm{HD}$, and we investigated their baseline characteristics (Supplementary Table 1). Among the 724 AF patients, there were 177 warfarin users (24.4\%). Comparisons between the two groups (warfarin users vs. warfarin non-users) showed that mean SBP $(133.7 \pm 14.6 \mathrm{mmHg}$ vs. $139.4 \pm 15.4 \mathrm{mmHg})$ and DBP $(75.6 \pm 10.2 \mathrm{mmHg}$ vs. $77.4 \pm 9.1 \mathrm{mmHg})$ were significantly lower among warfarin users than among nonusers. Moreover, serum albumin and creatinine levels were also significantly lower among warfarin users compared with non-users. However, there were significantly more cases of congestive heart failure, cerebrovascular disease, $\mathrm{DM}$, and stroke among warfarin users than among nonusers (Supplementary Table 1, 5).

We next performed multivariate Cox proportional regression analysis to investigate the impact of warfarin treatment. Use of warfarin was significantly associated with increased hemorrhagic stroke incidence (HR, 1.593; 95\% CI, 1.075-2.360; $\mathrm{p}=0.020$ ) relative to non-use, while there was no significant association between warfarin treatment and all-cause mortality, hospitalization, and ischemic stroke (Table 4).

We next performed Kaplan-Meier curve analysis for both warfarin user and non-user groups within the AF group for death, hospitalization, cerebral infarction, and cerebral hemorrhage. No statistically significant differences were observed in the four curves (Supplementary Fig. 2, available online).

\section{Discussion}

The prevalence of AF in Korea increased by 2.10 -fold from $0.73 \%$ in 2006 to $1.53 \%$ in 2015 . The annual trends of AF incidence were stable, with a 10 -year overall incidence of 1.77 per 1,000 person-years [10]. Unfortunately, there has been no data on the prevalence and incidence of AF in Korean ESRD patients. However, we identified the prevalence in Korea as $3.3 \%$ in this study. This rate is lower than we expected. As shown in an international study called the Dialysis Outcomes and Practice Patterns Study in which 12 countries participated, among the cross-section of HD patients, $12.5 \%$ of patients $(2,188$ out of 17,513$)$ had preexisting AF at baseline, compared with $5.6 \%$ in Japan and $24.7 \%$ in Belgium. The incidence of newly diagnosed AF

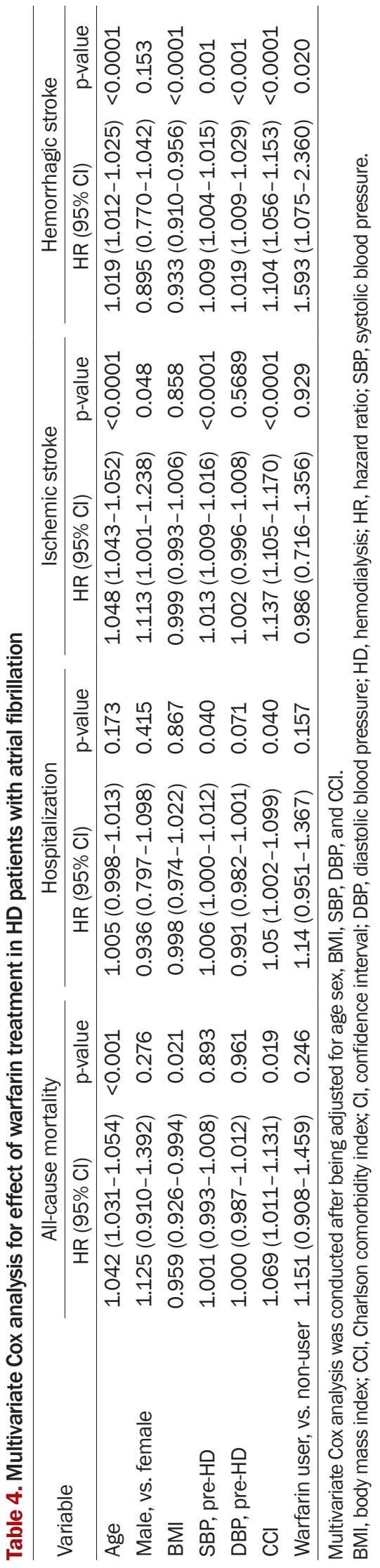

www.krcp-ksn.org 
during the follow-up period was 1.0 per 100 patient-years, compared with 0.5 in Japan and 3.0 in Sweden [11]. Since the adequacy assessment excludes people admitted during the period, AF prevalence may have been underestimated in our data.

We investigated whether AF was associated with a worse prognosis among HD patients using a large-scale, nationwide, population-based database. We noted that AF patients undergoing HD had significantly greater all-cause mortality, hospitalization, and hemorrhagic stroke, but not ischemic stroke. Moreover, we found that hemorrhagic stroke was more likely to occur among warfarin users among the AF patients undergoing HD compared with non-users, whereas warfarin treatment did not reduce or increase the risk of all-cause mortality, hospitalization, or ischemic stroke. Therefore, future studies should be performed with larger number of subjects in the warfarin-related cohort.

Several previous studies have demonstrated that AF patients on maintenance dialysis have a poor prognosis. In the RAKUEN (registry of atrial fibrillation in chronic kidney disease under hemodialysis from Niigata) study, which was a single-center, retrospective, observational study over a mean period of 36 months, $\mathrm{AF}$ was independently associated with death from any cause (HR, 1.69; 95\% CI, 1.04-2.74; $\mathrm{p}=$ 0.034 ), but not significantly associated with ischemic stroke or major bleeding events. Moreover, the findings suggested that AF had less impact on the risk of ischemic stroke in non-anticoagulated patients [12]. In a prospective study conducted by a Japanese group, the authors concluded that patients who had AF at the time of dialysis initiation showed high rates of mortality and cardiovascular or cerebrovascular disease [13]. The potential biologic mechanisms by which AF might cause death include thromboembolic events and worsening of heart failure (induced by tachycardia or possibly by beat-to-beat ventricular irregularity), although the absolute risk increase for heart failure was the highest among the outcomes, such as cardiovascular disease, renal disease, and stroke $[14,15]$. Data from a study conducted by Genovesi et al. [7] indicated that left atrial remodelingbecause of the presence of heart diseases-is likely to be accelerated by dialytic therapy and has an independent role in increasing the mortality rate. Consistent with these studies, we found that AF was significantly associated with an increased risk of death from any cause. However, we also found increased rates of hospitalization and hemorrhagic stroke in the AF group compared with the non-AF group. We could not determine why hemorrhagic stroke occurred more frequently among AF patients than among patients without AF, which was in contrast to the RAKUEN study. Thus, we investigated whether warfarin use would have an effect on hemorrhagic stroke incidence.

We found that warfarin use was significantly associated with an increased risk of hemorrhagic stroke among AF patients undergoing HD. The use of anticoagulation therapy for HD patients with AF is still controversial. In a retrospective cohort study, Kai et al. [16] concluded that warfarin use was associated with lower all-cause mortality and ischemic stroke risk, without significantly increasing the risk of bleeding in HD patients with AF. However, another study demonstrated a significant association between warfarin use and decreased risk of ischemic stroke but no association between warfarin use and death, hemorrhagic stroke, or gastrointestinal bleeding in intention-to-treat analyses (HR, 0.68; 95\% CI, 0.47-0.99). In contrast, in the astreated analyses, warfarin use was associated with reduced mortality (HR, 0.84; 95\% CI, 0.73-0.97) [17].

Some studies have reported findings that contrast with the above. In a prospective cohort study of Japanese HD patients with chronic sustained AF, warfarin use did not significantly reduce ischemic stroke events [18]. Moreover, in a metaanalysis performed by Lee et al. [19], which comprised eight studies with a total of 9,539 participants and 706 stroke events, warfarin use had a neutral effect on ischemic stroke or thromboembolism incidence among AF patients undergoing HD. The authors also indicated that warfarin use was associated with a higher risk of hemorrhagic stroke and bleeding and was associated with no effect on death.

The 2019 AHA/ACC/HRS guideline recommends that it might be reasonable to prescribe warfarin (international normalized ratio, 2.0-3.0) or apixaban for oral anticoagulation to patients with $\mathrm{AF}$ who have a $\mathrm{CHA}_{2} \mathrm{DS}_{2}$-VASc score of 2 or greater in men or 3 or greater in women and those who have end-stage chronic kidney disease (CKD; creatinine clearance of $<15 \mathrm{~mL} / \mathrm{min}$ ) or are on dialysis (Class of Recommendation IIb, Level of Evidence B-NR) [8]. In a large United States claims database analysis evaluating patients with $\mathrm{AF}$ and stage 4 or 5 CKD or undergoing hemolysis, rivaroxaban was associated with less major bleeding compared with warfarin in patients with severe kidney dysfunction (HR, 0.68; 95\% CI, 0.47-0.99) [20]. In 2018, Siontis et al. [21] published a 
study comparing apixaban to warfarin among patients with nonvalvular AF undergoing hemodialysis. No difference in the hazard of stroke or systemic embolism was revealed; however, apixaban was associated with a significantly lower risk of major bleeding (HR, 0.72; 95\% CI, 0.59-0.84; $\mathrm{p}<0.001$ ). Non-vitamin K oral anticoagulant, so-called NOAC, could possibly replace warfarin to lower the risk of bleeding.

This study had some limitations. The percentage of AF patients in our cohort was only 3.3\%. Under-diagnosis, missed diagnoses, and code input errors may have contributed to this low prevalence. In addition, all the diagnoses were based on ICD-10 codes, which do not differentiate between different types of AF (valvular vs. nonvalvular disease, chronic vs. paroxysmal, and fibrillation vs. flutter). Because the purpose of adequacy assessment is to qualify dialysis facilities themselves and acquire certification, there is some possibility of selection bias in favor of, for example, outpatients only or excluding patients with severe conditions. Furthermore, not all of the dialysis facilities in Korea were involved in the evaluation. As the national assessment for dialysis adequacy was undertaken from October to December 2013, its data collection period was three months which might reflect patient characteristics less well than one full year. Thus, the results should be interpreted with caution. The HAS-BLED score, which evaluates major bleeding risk among anticoagulation patients, was not collected or adjusted. Likewise, prescription of antiplatelet agents was not investigated. Since this study investigated the prescriptions of warfarin, the actual dose and adherence may differ among patients. Due to the limitations of detailed investigation in a retrospective study, it was difficult to clarify the successive relationship between warfarin and stroke.

Nevertheless, the strength of this study is that, as HIRA is a national system, our study included a large pool of subjects (a total of 21,839 patients). Furthermore, the followup duration was longer than in other previous studies. Additionally, as this study targeted only Korean patients, we generated novel data for this particular ethnic group, which can be compared with other groups.

In conclusion, our analysis showed that AF could be an independent risk factor for adverse clinical outcomes among HD patients. However, warfarin administration did not decrease the incidence of ischemic stroke, mortality, or hospitalization but was associated with an increased risk of hemorrhagic stroke among HD patients. Our study suggests that physicians should specifically assess for AF, and they should be more cautious with AF patients when they consider prescribing warfarin.

\section{Conflicts of interest}

All authors have no conflicts of interest to declare.

\section{Funding}

This work was supported by an Ewha Womans University Research Grant in 2018.

\section{Acknowledgments}

The authors participated in the Joint Project on Quality Assessment Research, and HIRA collected and provided the claims data and quality assessment data to the authors.

\section{Authors' contributions}

Conceptualization: YK, HJO, DRR

Data curation: HYC, YEK, JHS, EMW, KHY

Formal analysis: YK, HJO, DRR

Investigation: DRR

Methodology: DRR

Project administration: HJO, DRR

Visualization: YK

Writing-original draft: YK, HJO

Writing-review \& editing: All authors

All authors read and approved the final manuscript.

\section{ORCID}

Yeunmi Kang, https://orcid.org/0000-0002-8475-2255

Hyung Yun Choi, https://orcid.org/0000-0001-8496-6723

Young Eun Kwon, https://orcid.org/0000-0002-8370-1333

Ji Hyeon Shin, https://orcid.org/0000-0001-8915-6885

Eun Mi Won, https://orcid.org/0000-0001-6213-394X

Ki Hwa Yang, https://orcid.org/0000-0003-0134-3059

Hyung Jung Oh, https://orcid.org/0000-0002-4281-696X

Dong-Ryeol Ryu, https://orcid.org/0000-0002-5309-7606 


\section{References}

1. Königsbrügge O, Posch F, Antlanger M, et al. Prevalence of atrial fibrillation and antithrombotic therapy in hemodialysis patients: cross-sectional results of the Vienna In-Vestigation of AtriaL Fibrillation and Thromboembolism in Patients on HemoDIalysis (VIVALDI). PLoS One 2017;12:e0169400.

2. Ball J, Carrington MJ, McMurray JJ, Stewart S. Atrial fibrillation: profile and burden of an evolving epidemic in the 21st century. Int J Cardiol 2013;167:1807-1824.

3. Saran R, Robinson B, Abbott KC, et al. US renal data system 2016 annual data report: epidemiology of kidney disease in the United States. Am J Kidney Dis 2017;69(3 Suppl 1):A7-A8.

4. Goldstein BA, Arce CM, Hlatky MA, Turakhia M, Setoguchi S, Winkelmayer WC. Trends in the incidence of atrial fibrillation in older patients initiating dialysis in the United States. Circulation 2012;126:2293-2301.

5. Falk RH. Etiology and complications of atrial fibrillation: insights from pathology studies. Am J Cardiol 1998;82:10N-17N.

6. Buiten MS, de Bie MK, Rotmans JI, et al. The dialysis procedure as a trigger for atrial fibrillation: new insights in the development of atrial fibrillation in dialysis patients. Heart 2014;100:685-690.

7. Genovesi S, Vincenti A, Rossi E, et al. Atrial fibrillation and morbidity and mortality in a cohort of long-term hemodialysis patients. Am J Kidney Dis 2008;51:255-262.

8. Herzog CA, Asinger RW, Berger AK, et al. Cardiovascular disease in chronic kidney disease. A clinical update from Kidney Disease: Improving Global Outcomes (KDIGO). Kidney Int 2011;80:572586.

9. Writing Group Members; January CT, Wann LS, et al. 2019 AHA/ ACC/HRS focused update of the 2014 AHA/ACC/HRS guideline for the management of patients with atrial fibrillation: a report of the American College of Cardiology/American Heart Association Task Force on clinical practice guidelines and the Heart Rhythm Society. Heart Rhythm 2019;16:e66-e93.

10. Joung B, Lee JM, Lee KH, et al. 2018 Korean guideline of atrial fibrillation management. Korean Circ J 2018;48:1033-1080.

11. Wizemann V, Tong L, Satayathum S, et al. Atrial fibrillation in hemodialysis patients: clinical features and associations with anticoagulant therapy. Kidney Int 2010;77:1098-1106.

12. Mitsuma W, Matsubara T, Hatada K, et al. Atrial fibrillation had less impact on the risk of ischemic stroke in nonanticoagulated patients undergoing hemodialysis: insight from the RAKUEN study. Intern Med 2018;57:2295-2300.

13. Tanaka A, Inaguma D, Shinjo H, Murata M, Takeda A; Aichi Cohort Study of Prognosis in Patients Newly Initiated into Dialysis (AICOPP) Study Group. Presence of atrial fibrillation at the time of dialysis initiation is associated with mortality and cardiovascular events. Nephron 2016;132:86-92.

14. Leong DP, Eikelboom JW, Healey JS, Connolly SJ. Atrial fibrillation is associated with increased mortality: causation or association? Eur Heart J 2013;34:1027-1030.

15. Odutayo A, Wong CX, Hsiao AJ, Hopewell S, Altman DG, Emdin CA. Atrial fibrillation and risks of cardiovascular disease, renal disease, and death: systematic review and meta-analysis. $B M J$ 2016;354:i4482.

16. Kai B, Bogorad Y, Nguyen LN, et al. Warfarin use and the risk of mortality, stroke, and bleeding in hemodialysis patients with atrial fibrillation. Heart Rhythm 2017;14:645-651.

17. Shen JI, Montez-Rath ME, Lenihan CR, Turakhia MP, Chang TI, Winkelmayer WC. Outcomes after warfarin initiation in a cohort of hemodialysis patients with newly diagnosed atrial fibrillation. Am J Kidney Dis 2015;66:677-688.

18. Wakasugi M, Kazama JJ, Tokumoto A, et al. Association between warfarin use and incidence of ischemic stroke in Japanese hemodialysis patients with chronic sustained atrial fibrillation: a prospective cohort study. Clin Exp Nephrol 2014;18:662-669.

19. Lee M, Saver JL, Hong KS, et al. Warfarin use and risk of stroke in patients with atrial fibrillation undergoing hemodialysis: a meta-analysis. Medicine (Baltimore) 2016;95:e2741.

20. Coleman CI, Kreutz R, Sood NA, et al. Rivaroxaban versus warfarin in patients with nonvalvular atrial fibrillation and severe kidney disease or undergoing hemodialysis. Am J Med 2019;132:1078-1083.

21. Siontis KC, Zhang X, Eckard A, et al. Outcomes associated with apixaban use in patients with end-stage kidney disease and atrial fibrillation in the United States. Circulation 2018;138:15191529. 